\title{
RIGIDITY OF SCATTERING LENGTHS AND TRAVELLING TIMES FOR DISJOINT UNIONS OF STRICTLY CONVEX BODIES
}

\author{
LYLE NOAKES AND LUCHEZAR STOYANOV \\ (Communicated by Yingfei Yi)
}

\begin{abstract}
Obstacles $K$ and $L$ in $\mathbb{R}^{d}(d \geq 2)$ are considered that are finite disjoint unions of strictly convex domains with $C^{3}$ boundaries. We show that if $K$ and $L$ have (almost) the same scattering length spectrum, or (almost) the same travelling times, then $K=L$.
\end{abstract}

\section{INTRODUCTION}

Let $K$ be a compact subset of $\mathbb{R}^{d}(d \geq 2)$ with $C^{3}$ boundary $\partial K$ such that $\Omega_{K}=\overline{\mathbb{R}^{d} \backslash K}$ is connected. A scattering ray $\gamma$ in $\Omega_{K}$ is an unbounded in both directions generalized geodesic (in the sense of Melrose and Sjöstrand [MS1, MS2]). If $K$ is a finite disjoint union of convex domains, then the scattering rays in $\Omega_{K}$ are simply billiard trajectories with finitely many common points with $\partial K$. This article concerns two types of problems related to recovering information about the obstacle $K$ from certain measurements of scattering rays in the exterior of $K$. These problems have similarities with various problems on metric rigidity in Riemannian geometry - see [SU], SUV] and the references there for more information.

1.1. The scattering length spectrum. The first type of problems deal with the so-called scattering length spectrum (SLS). Given a scattering ray $\gamma$ in $\Omega_{K}$, denote by $T_{\gamma}$ the sojourn time of $\gamma$ (cf. Sect. 2). If $\omega \in \mathbf{S}^{d-1}$ is the incoming direction of $\gamma$ and $\theta \in \mathbf{S}^{d-1}$ its outgoing direction, $\gamma$ will be called an $(\omega, \theta)$-ray. The scattering length spectrum of $K$ is defined to be the family of sets of real numbers $\mathcal{S} L_{K}=\left\{\mathcal{S} L_{K}(\omega, \theta)\right\}_{(\omega, \theta)}$ where $(\omega, \theta)$ runs over $\mathbf{S}^{d-1} \times \mathbf{S}^{d-1}$ and $\mathcal{S} L_{K}(\omega, \theta)$ is the set of sojourn times $T_{\gamma}$ of all $(\omega, \theta)$-rays $\gamma$ in $\Omega_{K}$. It is known (cf. [St3]) that for $d \geq 3, d$ odd, and $C^{\infty}$ boundary $\partial K$, we have $\mathcal{S} L_{K}(\omega, \theta)=\operatorname{sing} \operatorname{supp} s_{K}(t, \theta, \omega)$ for almost all $(\omega, \theta)$. Here $s_{K}$ is the scattering kernel related to the scattering operator for the wave equation in $\mathbb{R} \times \Omega_{K}$ with Dirichlet boundary condition on $\mathbb{R} \times \partial \Omega_{K}$ (cf. [LP1], [M]). Following [St4, we will say that two obstacles $K$ and $L$ have almost the same $S L S$ if there exists a subset $\mathcal{R}$ of full Lebesgue measure in $\mathbf{S}^{d-1} \times \mathbf{S}^{d-1}$ such that $\mathcal{S} L_{K}(\omega, \theta)=\mathcal{S} L_{L}(\omega, \theta)$ for all $(\omega, \theta) \in \mathcal{R}$.

It is a natural and rather important problem in inverse scattering by obstacles to get information about the obstacle $K$ from its SLS. It is known that various kinds of information about $K$ can be recovered from its SLS, and for some classes of obstacles $K$ is completely recoverable (see e.g. [Ma], [MaR], [LP2], St2], St4]),

Received by the editors February 7, 2014 and, in revised form, April 28, 2014.

2010 Mathematics Subject Classification. Primary 37D20, 37D40, 53D25, 58J50. 
for example star-shaped obstacles are in this class. However, as an example of M. Livshits shows (cf. Ch. 5 in [M]; see also Figure 1), in general $\mathcal{S} L_{K}$ does not determine $K$ uniquely.

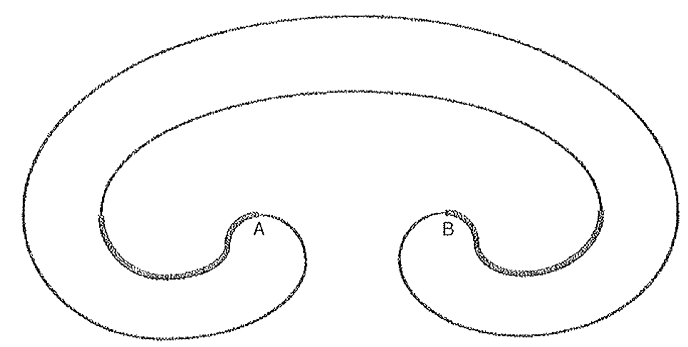

Figure 1. Livshits' example (adapted from Ch. 5 of [M]): the internal upper part of the figure is half an ellipse with foci $A$ and $B$. A ray entering the interior of the ellipse between the foci must exit between the foci after reflection. So, no scattering ray has a common point with the bold parts of the boundary.

1.2. Travelling times. The second type of problems deal with travelling times. Let $\mathcal{O}$ be a large ball in $\mathbb{R}^{d}$ containing $K$ in its interior and set $S_{0}:=\partial \mathcal{O}$. For any pair of points $x, y \in S_{0}$ consider the scattering rays $\gamma$ incoming through the point $x$ and outgoing through the point $y$. Such rays will be called $(x, y)$-geodesics in $\Omega_{K}$. Given such $\gamma$, let $t_{\gamma}$ be the length of the part of $\gamma$ from $x$ to $y$. Let $\mathcal{T}_{K}(x, y)$ the set of travelling times $t_{\gamma}$ of all $(x, y)$-geodesic in $\Omega_{K}$. If $K$ and $L$ are two obstacles contained in the interior of $S_{0}$, we will say that $K$ and $L$ have almost the same travelling times if $\mathcal{T}_{K}(x, y)=\mathcal{T}_{L}(x, y)$ for almost all $(x, y) \in S_{0} \times S_{0}$ (with respect to the Lebesgue measure on $S_{0} \times S_{0}$ ). Our second type of problems concern getting information about the obstacle $K$ from its travelling times.

1.3. Unions of convex bodies. For either kind of problems, we consider obstacles $K$ of the form

$$
K=K_{1} \cup K_{2} \cup \ldots \cup K_{k_{0}},
$$

where $K_{i}$ are strictly convex disjoint domains in $\mathbb{R}^{d}(d \geq 2)$ with $C^{3}$ smooth boundaries $\partial K_{i}$. In this case the so-called generalized Hamiltonian (or bicharacteristic) flow $\mathcal{F}_{t}^{(K)}: S^{*}\left(\Omega_{K}\right) \longrightarrow S^{*}\left(\Omega_{K}\right)$ (see Sect. 2) coincides with the billiard flow, so it is easier to deal with.

A point $\sigma=(x, \omega) \in S^{*}\left(\Omega_{K}\right)$ is called non-trapped if both curves $\left\{\operatorname{pr}_{1}\left(\mathcal{F}_{t}^{(K)}(\sigma)\right)\right.$ : $t \leq 0\}$ and $\left\{\operatorname{pr}_{1}\left(\mathcal{F}_{t}^{(K)}(\sigma)\right): t \geq 0\right\}$ in $\Omega_{K}$ are unbounded. Otherwise $\sigma$ is called a trapped point. Here we use the notation $\operatorname{pr}_{1}(y, \eta)=y$ and $\operatorname{pr}_{2}(y, \eta)=\eta$. Denote by $\operatorname{Trap}\left(\Omega_{K}\right)$ the set of all trapped points. It is well known that in general $\operatorname{Trap}\left(\Omega_{K}\right)$ may have positive Lebesgue measure and a non-empty interior in $S^{*}\left(\Omega_{K}\right)$ (see e.g. Livshits' example). Set $\dot{T}^{*}\left(\Omega_{K}\right)=T^{*}\left(\Omega_{K}\right) \backslash\{0\}$.

Definition 1.1. Let $K, L$ be two obstacles in $\mathbb{R}^{d}$. We will say that $\Omega_{K}$ and $\Omega_{L}$ have conjugate flows if there exists a homeomorphism

$$
\Phi: \dot{T}^{*}\left(\Omega_{K}\right) \backslash \operatorname{Trap}\left(\Omega_{K}\right) \longrightarrow \dot{T}^{*}\left(\Omega_{L}\right) \backslash \operatorname{Trap}\left(\Omega_{L}\right),
$$


which has the following properties: (i) $\Phi$ defines a symplectic map on an open dense subset of $\dot{T}^{*}\left(\Omega_{K}\right) \backslash \operatorname{Trap}\left(\Omega_{K}\right)$, (ii) $\Phi\left(S^{*}\left(\Omega_{K}\right) \backslash \operatorname{Trap}\left(\Omega_{K}\right)\right)=S^{*}\left(\Omega_{L}\right) \backslash \operatorname{Trap}\left(\Omega_{L}\right)$, (iii) $\mathcal{F}_{t}^{(L)} \circ \Phi=\Phi \circ \mathcal{F}_{t}^{(K)}$ for all $t \in \mathbb{R}$ and $\Phi=$ id on $\dot{T}^{*}\left(\mathbb{R}^{d} \backslash \mathcal{O}\right) \backslash \operatorname{Trap}\left(\Omega_{K}\right)=$ $\dot{T}^{*}\left(\mathbb{R}^{d} \backslash \mathcal{O}\right) \backslash \operatorname{Trap}\left(\Omega_{L}\right)$.

It is known that for $K, L$ in a very large (generic) class of obstacles in $\mathbb{R}^{d}(d \geq 2)$, if $K$ and $L$ have almost the same SLS or almost the same travelling times, then $\Omega_{K}$ and $\Omega_{L}$ have conjugate flows ( $[\mathrm{St} 4$ ] and $[\mathrm{NS}$; ; see Sect. 2 below where these results are given in full details). In this paper we prove

Theorem 1.2. Let $d \geq 2$ and let each of the obstacles $K$ and $L$ be a finite disjoint union of strictly convex domains in $\mathbb{R}^{d}$ with $C^{3}$ boundaries. If $\Omega_{K}$ and $\Omega_{L}$ have conjugate flows, then $K=L$.

For the case where $\partial K$ and $\partial L$ are real analytic this was proved in St2]. As an immediate consequence of Theorem 1.2 and results in St4] and [NS], one gets the following.

Corollary 1.3. Assume that $K$ and $L$ are obstacles in $\mathbb{R}^{d}(d \geq 2)$ and each of them is a finite disjoint union of strictly convex domains with $C^{3}$ boundaries. If $K$ and $L$ have almost the same scattering length spectrum, or $K$ and $L$ have almost the same travelling times, then $K=L$.

We remark that the above results are non-trivial. Indeed when $K$ has a large number of connected components and they are densely packed (imagine the molecules of a gas in a container), then there are a great number of billiard trajectories with large numbers of reflections (and possibly with many tangencies) in the exterior of $K$. Moreover, in such cases many connected components of $K$ can only be reached by billiard trajectories having many reflections. So, being able to completely recover the obstacle $K$ by measuring sojourn times only, or travelling times only, is far from being a trivial matter. The assumption that $\partial K$ and $\partial L$ are $C^{3}$ smooth is required in order to be able to use some of the results in St2] and St4.

\section{Preliminaries}

We refer the reader to MS1, MS2 (or Sect. 24.3 in [H]) for the definition of the generalized Hamiltonian (bicharacteristic) flow on a symplectic manifold with boundary. In the case of scattering by an obstacle $K$ this is the generalized geodesic flow $\mathcal{F}_{t}^{(K)}: \dot{T}_{b}^{*}\left(\Omega_{K}\right)=T_{b}^{*}\left(\Omega_{K}\right) \backslash\{0\} \longrightarrow \dot{T}_{b}^{*}\left(\Omega_{K}\right)$ generated by the principal symbol of the wave operator in $\mathbb{R} \times \Omega_{K}$. Here $T_{b}^{*}\left(\Omega_{K}\right)=T^{*}\left(\Omega_{K}\right) / \sim$ is the quotient space with respect to the following equivalence relation on $T^{*}\left(\Omega_{K}\right):(x, \xi) \sim(y, \eta)$ iff $x=y$ and either $\xi=\eta$ or $x=y \in \partial K$ and $\xi$ and $\eta$ are symmetric with respect to the tangent plane to $\partial K$ at $x$. The image $S_{b}^{*}\left(\Omega_{K}\right)$ of the unit cosphere bundle $S^{*}\left(\Omega_{K}\right)$ under the natural projection is invariant with respect to $\mathcal{F}_{t}^{(K)}$. For simplicity of notation the subscript $b$ will be suppressed, and it will be clear from the context exactly which second component we have in mind.

In general $\mathcal{F}_{t}^{(K)}$ is not a flow in the usual sense of dynamical systems, since there may exist different integral curves issued from the same point of the phase space (see [T]). Let $\mathcal{K}$ be the class of obstacles that have the following property: for each $(x, \xi) \in \dot{T}^{*}(\partial K)$ if the curvature of $\partial K$ at $x$ vanishes of infinite order in direction $\xi$, then all points $(y, \eta)$ sufficiently close to $(x, \xi)$ are diffractive (roughly speaking, 
this means that $\partial K$ is convex at $y$ in the direction of $\eta$ ). It follows that for $K \in \mathcal{K}$ the flow $\mathcal{F}_{t}^{(K)}$ is well-defined and continuous ( $\mathrm{MS} 2$ ).

We now describe the relevant results from St4 and NS used in the proof of Corollary 1.3. Given $\xi \in \mathbf{S}^{d-1}$ denote by $Z_{\xi}$ the hyperplane in $\mathbb{R}^{d}$ orthogonal to $\xi$ and tangent to $\mathcal{O}$ such that $\mathcal{O}$ is contained in the open half-space $R_{\xi}$ determined by $Z_{\xi}$ and having $\xi$ as an inner normal. For an $(\omega, \theta)$-ray $\gamma$ in $\Omega$, the sojourn time $T_{\gamma}$ of $\gamma$ is defined by $T_{\gamma}=T_{\gamma}^{\prime}-2 a$, where $T_{\gamma}^{\prime}$ is the length of that part of $\gamma$ which is contained in $R_{\omega} \cap R_{-\theta}$ and $a$ is the radius of the ball $\mathcal{O}$. It is known (cf. [G]) that this definition does not depend on the choice of the ball $\mathcal{O}$. Following [PS2], given $\sigma=(x, \xi) \in S^{*}\left(\Omega_{K}\right)$ so that $\gamma_{K}(\sigma)=\left\{\operatorname{pr}_{1}\left(\mathcal{F}_{t}^{(K)}(\sigma)\right): t \in \mathbb{R}\right\}$ is a simply reflecting ray, i.e. it has no tangencies to $\partial K$, we will say that $\gamma_{K}(\sigma)$ is non-degenerate if for every $t>>0$ the map $\mathbb{R}^{d} \ni y \mapsto \operatorname{pr}_{2}\left(\mathcal{F}_{t}^{(K)}(y, \xi)\right) \in \mathbf{S}^{d-1}$ is a submersion at $y=x$, i.e. its differential at $y=x$ has rank $d-1$. Set $S_{+}^{*}\left(S_{0}\right)=\left\{(x, u): x \in S_{0}, u \in\right.$ $\left.\mathbf{S}^{d-1},\left\langle\nu_{x}, u\right\rangle<0\right\}$, where $\nu_{x}$ is the outward unit normal to $S_{0}$ at $x$. Denote by $\mathcal{K}_{0}$ the class of all obstacles $K \in \mathcal{K}$ contained in $\mathcal{O}$ satisfying the following nondegeneracy conditions: $\gamma_{K}(\sigma)$ is a non-degenerate simply reflecting ray for almost all $\sigma \in S_{+}^{*}\left(S_{0}\right) \backslash \operatorname{Trap}\left(\Omega_{K}\right)$ such that $\gamma_{K}(\sigma) \cap \partial K \neq \emptyset$, and $\partial K$ does not contain non-trivial open flat subsets (i.e. open subsets where the curvature is zero at every point). It can be derived without much difficulty from [PS1] (see Ch. 3 there) that $\mathcal{K}_{0}$ is of second Baire category in $\mathcal{K}$ with respect to the $C^{\infty}$ Whitney topology in $\mathcal{K}$. That is, for every $K \in \mathcal{K}$, applying suitable arbitrarily small $C^{\infty}$ deformations to $\partial K$, one gets obstacles from the class $\mathcal{K}_{0}$ and most deformations have this property.

Theorem 2.1 ([St4]). If the obstacles $K, L \in \mathcal{K}_{0}$ have almost the same $S L S$, then $\Omega_{K}$ and $\Omega_{L}$ have conjugate flows. Conversely, if $K, L \in \mathcal{K}_{0}$ have conjugate flows, then $K$ and $L$ have the same $S L S$.

There is a similar result for the travelling times spectrum. Consider the crosssectional map $\mathcal{P}_{K}: S_{+}^{*}\left(S_{0}\right) \backslash \operatorname{Trap}\left(\Omega_{K}\right) \longrightarrow S^{*}\left(S_{0}\right)$ defined by the shift along the flow $\mathcal{F}_{t}^{(K)}$. Let $\gamma$ be a $\left(x_{0}, y_{0}\right)$-geodesic in $\Omega_{K}$ for some $x_{0}, y_{0} \in S_{0}$, which is a simply reflecting ray. Let $\omega_{0} \in \mathbf{S}^{d-1}$ be the (incoming) direction of $\gamma$ at $x_{0}$. We will say that $\gamma$ is regular if the differential of the map $\mathbf{S}^{d-1} \ni \omega \mapsto \mathcal{P}_{K}\left(x_{0}, \omega\right) \in S_{0}$ is a submersion at $\omega=\omega_{0}$, i.e. its differential at that point has rank $d-1$. Denote by $\mathcal{L}_{0}$ the class of all obstacles $K \in \mathcal{K}$ such that $\partial K$ does not contain non-trivial open flat subsets and $\gamma_{K}(x, u)$ is a regular simply reflecting ray for almost all $(x, u) \in S_{+}^{*}\left(S_{0}\right)$ such that $\gamma(x, u) \cap \partial K \neq \emptyset$. Using an argument from Ch. 3 in PS1 one can show that $\mathcal{L}_{0}$ is of second Baire category in $\mathcal{K}$ with respect to the $C^{\infty}$ Whitney topology in $\mathcal{K}$. That is, generic obstacles $K \in \mathcal{K}$ belong to the class $\mathcal{L}_{0}$.

Theorem 2.2 ([NS]). If the obstacles $K, L \in \mathcal{L}_{0}$ have almost the same travelling times, then $\Omega_{K}$ and $\Omega_{L}$ have conjugate flows. Conversely, if $K, L \in \mathcal{L}_{0}$ have conjugate flows, then $K$ and $L$ have the same travelling times.

Next, we describe four propositions from [St2] and [St4 that are needed in the proof of Theorem 1.2. In what follows we assume

Hypothesis SCC. $K$ and $L$ are finite disjoint unions of strictly convex domains in $\mathbb{R}^{d}(d \geq 2)$ with $C^{3}$ boundaries, and with conjugate generalized geodesic flows.

Given $\sigma \in S^{*}\left(\Omega_{K}\right)$ denote $\gamma_{K}^{+}(\sigma):=\left\{\operatorname{pr}_{1}\left(\mathcal{F}_{t}^{(K)}(\sigma)\right): t \geq 0\right\}$. 
Proposition 2.3 ([St2]). (a) There exists a countable family $\left\{M_{i}\right\}=\left\{M_{i}^{(K)}\right\}$ of codimension 1 submanifolds of $S_{+}^{*}\left(S_{0}\right) \backslash \operatorname{Trap}\left(\Omega_{K}\right)$ such that every $\sigma \in S_{+}^{*}\left(S_{0}\right) \backslash$ $\left(\operatorname{Trap}\left(\Omega_{K}\right) \cup_{i} M_{i}\right)$ generates a simply reflecting ray in $\Omega_{K}$. Moreover the family $\left\{M_{i}\right\}$ is locally finite, that is any compact subset of $S_{+}^{*}\left(S_{0}\right) \backslash \operatorname{Trap}\left(\Omega_{K}\right)$ has common points with only finitely many of the submanifolds $M_{i}$.

(b) There exists a countable locally finite family $\left\{R_{i}\right\}$ of codimension 2 smooth submanifolds of $S_{+}^{*}\left(S_{0}\right)$ such that for any $\sigma \in S_{+}^{*}\left(S_{0}\right) \backslash\left(\cup_{i} R_{i}\right)$ the trajectory $\gamma_{K}(\sigma)$ has at most one tangency to $\partial K$.

(c) There exists a countable family $\left\{Q_{i}\right\}$ of codimension 2 smooth submanifolds of $S_{\partial K}^{*}\left(\Omega_{K}\right)$ such that for any $\sigma \in S_{+}^{*}\left(S_{0}\right) \backslash\left(\cup_{i} Q_{i}\right)$ the trajectory $\gamma_{K}(\sigma)$ has at most one tangency to $\partial K$.

It follows from the conjugacy of flows and Proposition 4.3 in St4 that the submanifolds $M_{i}$ are the same for $K$ and $L$, i.e. $M_{i}^{(K)}=M_{i}^{(L)}$ for all $i$.

Remark. Different submanifolds $M_{i}$ and $M_{j}$ may have common points (these generate rays with more than one tangency to $\partial K$ ) and in general are not transversal to each other. However, as we see from part (b), if $M_{i} \neq M_{j}$ and $\sigma \in M_{i} \cap M_{j}$, then locally near $\sigma, M_{i} \neq M_{j}$, i.e. there exist points in $M_{i} \backslash M_{j}$ arbitrarily close to $\sigma$.

For the present case we also have some information about the size of the set $\operatorname{Trap}\left(\Omega_{K}\right)$.

Proposition 2.4 ([St2]). Let $d \geq 2$ and let $K$ have the form (1.1). Then $S_{+}^{*}\left(S_{0}\right) \backslash$ $\operatorname{Trap}\left(\Omega_{K}\right)$ is an arc connected open dense subset of $S_{+}^{*}\left(S_{0}\right)$.

Proof. This is essentially proved in St2] assuming $d \geq 3$, however a small modification of the argument works for $d=2$ as well. We sketch it here for completeness. So, assume that $d \geq 2$. Clearly $S_{+}^{*}\left(S_{0}\right) \backslash \operatorname{Trap}\left(\Omega_{K}\right)$ is open in $S_{+}^{*}\left(S_{0}\right)$.

Consider an arbitrary $\sigma_{0}^{\prime}=\left(x_{0}^{\prime}, \xi_{0}^{\prime}\right) \in S_{+}^{*}\left(S_{0}\right) \backslash \operatorname{Trap}\left(\Omega_{K}\right)$. We will find a continuous curve $\sigma^{\prime}(t)$ in $S^{*}\left(\mathbb{R}^{d} \backslash \mathcal{O}\right) \backslash \operatorname{Trap}\left(\Omega_{K}\right)$ such that $\sigma^{\prime}(0)=\sigma_{0}^{\prime}$ and $\sigma^{\prime}(1)$ generates a free trajectory in $S^{*}\left(\mathbb{R}^{n} \backslash \mathcal{O}\right)$, i.e. a trajectory without any reflections at $\partial K$. We will assume that the trajectory $\gamma_{K}^{+}\left(\sigma_{0}\right)$ has a common point with $K$; otherwise there is nothing to prove.

There exists a strictly convex smooth hypersurface $X$ in $\mathbb{R}^{n} \backslash \mathcal{O}$ with a continuous unit normal field $\nu_{X}$ such that for some $x_{0} \in X$ and $t>0$, we have $x_{0}^{\prime}=x_{0}+t \nu_{X}\left(x_{0}\right)$ and $\nu_{X}\left(x_{0}\right)=\xi_{0}^{\prime}$, and there exists $x_{1} \in X$ such that the ray $\left\{x_{1}+t \nu_{X}\left(x_{1}\right): t>0\right\}$ has no common points with $K-$ e.g. take the boundary sphere $X$ of a very large ball in $\mathbb{R}^{d} \backslash \mathcal{O}$ with exterior unit normal field $\nu_{X}$. Clearly it is enough to construct a $C^{1}$ curve $\sigma(t)$ in $S^{*}(X) \backslash \operatorname{Trap}\left(\Omega_{K}\right)$ such that $\sigma(0)=\left(x_{0}, \nu_{X}\left(x_{0}\right)\right)$ and $\sigma(1)=$ $\left(x_{1}, \nu_{X}\left(x_{1}\right)\right)$.

Set $\widetilde{X}=\left\{\left(x, \nu_{X}(x)\right): x \in X\right\}$. Considering trajectories $\gamma_{K}^{+}(\sigma)$ with $\sigma \in \widetilde{X}$ and using the strict convexity of $X$ will allow us to use the strong hyperbolicity properties of the billiard flow in $\Omega_{K}$ ([Si1], Si2]; see also [St1]).

Take a very large open ball $U_{1}$ that contains the orthogonal projection of the convex hull $\hat{K}$ of $K$ onto $X$ and the point $x_{1}$ as well. Since $\operatorname{Trap}\left(\Omega_{K}\right) \cap S^{*}\left(U_{1}\right)$ is compact and $\sigma_{0}=\left(x_{0}, \nu_{X}\left(x_{0}\right) \notin \operatorname{Trap}\left(\Omega_{K}\right)\right.$, there exists an open connected neighbourhood $V_{0}$ of $\sigma_{0}$ in $S^{*}\left(U_{1}\right)$ with $V_{0} \cap \operatorname{Trap}\left(\Omega_{K}\right)=\emptyset$. 
It follows from Proposition 2.3(b) that there exists a countable family $\left\{Q_{i}^{\prime}\right\}$ of smooth codimension 2 submanifolds of $S^{*}\left(U_{1}\right)$ such that for any $\sigma \in S^{*}\left(U_{1}\right) \backslash$ $\left(\bigcup_{i} Q_{i}^{\prime}\right)$, the trajectory $\gamma_{K}(\sigma)$ has at most one tangency to $\partial K$. The submanifolds $Q_{i}^{\prime}$ are obtained from the submanifolds $Q_{i}$ in Proposition 2.3(b) by translation along the second (vector) component, so, locally they are invariant under the flow $\mathcal{F}_{t}^{(K)}$.

Using Thom's Transversality Theorem (cf. e.g. Hi]), and applying an arbitrarily small in the $C^{3}$ Whitney topology deformation to $X$, as in the proof of Proposition 5.1 in St2, without loss of generality, we may assume that $\widetilde{X}$ is transversal to each of the submanifolds $Q_{i}^{\prime}$. When $d=2$, this simply means that $\widetilde{X} \cap Q_{i}^{\prime}$ is a discrete subset of $\tilde{X}$. When $d \geq 3, \tilde{X} \cap Q_{i}^{\prime}$ is a submanifold of $\widetilde{X}$ with $\operatorname{dim}\left(\tilde{X} \cap Q_{i}\right)=d-3$. By the Sum Theorem for the topological dimension dim (cf. e.g. Theorem 15 in $[\mathrm{F}])$, for $X^{\prime}=\widetilde{X} \cap\left(\bigcup_{i} Q_{i}\right)$ we get $\operatorname{dim}\left(X^{\prime}\right) \leq d-3$.

Next, denote by $X_{0}$ the set of those $\sigma \in \widetilde{X} \cap \operatorname{Trap}\left(\Omega_{K}\right)$ such that the trajectory $\gamma_{K}^{+}(\sigma)$ has no tangencies to $\partial K$. Given integers $p, q$ such that $q \in\left\{1, \ldots, k_{0}\right\}$ and $p \geq 1$, denote by $X(p, q)$ the set of those $\sigma \in \tilde{X} \cap \operatorname{Trap}\left(\Omega_{K}\right)$ such that $\gamma_{K}^{+}(\sigma)$ has exactly one tangency to $\partial K$ which is its $p$ th reflection point and it belongs to $\partial K_{q}$. As in the proof of Proposition 5.1 in St2] (see also the proof of Lemma 3.1 below where we repeat this argument), it follows that each of the subspaces $X_{0}$ and $X(p, q)$ of $\tilde{X}$ has topological dimension 0. Assuming $d \geq 3$, the Sum Theorem for topological dimension (cf. Theorem 15 in $[\mathrm{F}$ ) shows that

$$
\operatorname{dim}\left(\tilde{X} \cap \operatorname{Trap}\left(\Omega_{K}\right)\right) \leq \operatorname{dim}\left(X^{\prime} \cup X_{0} \cup \bigcup_{p, q} X(p, q)\right) \leq d-3 .
$$

In the case $d=2$ we simply have $\operatorname{dim}\left(\widetilde{X} \cap \operatorname{Trap}\left(\Omega_{K}\right)\right)=0$. In both cases a theorem by Mazurkiewicz (see e.g. Theorem 25 in $[\mathrm{F}]$ ) implies that $\widetilde{X} \backslash \operatorname{Trap}\left(\Omega_{K}\right)$ is arc connected. Thus, there exists a $C^{1}$ curve $\sigma(t)$ in $\widetilde{X} \backslash \operatorname{Trap}\left(\Omega_{K}\right)$ (and so in $\left.S^{*}(X) \backslash \operatorname{Trap}\left(\Omega_{K}\right)\right)$ such that $\sigma(0)=\left(x_{0}, \nu_{X}\left(x_{0}\right)\right)$ and $\sigma(1)=\left(x_{1}, \nu_{X}\left(x_{1}\right)\right)$. This proves that $S_{+}^{*}\left(S_{0}\right) \backslash \operatorname{Trap}\left(\Omega_{K}\right)$ is arc connected.

It remains to show that $S_{+}^{*}\left(S_{0}\right) \backslash \operatorname{Trap}\left(\Omega_{K}\right)$ is dense in $S_{+}^{*}\left(S_{0}\right)$. Consider an arbitrary $\sigma_{0}^{\prime}=\left(x_{0}^{\prime}, \xi_{0}^{\prime}\right) \in S_{+}^{*}\left(S_{0}\right)$ and construct $X$ and $\widetilde{X}$ as above. Then the above argument shows that $\operatorname{dim}\left(\tilde{X} \cap \operatorname{Trap}\left(\Omega_{K}\right)\right) \leq d-3$ when $d \geq 3$, and $\operatorname{dim}\left(\tilde{X} \cap \operatorname{Trap}\left(\Omega_{K}\right)\right)=0$ when $d=2$. In both cases there exist points $\sigma \in$ $\tilde{X} \backslash \operatorname{Trap}\left(\Omega_{K}\right)$ arbitrarily close to $\sigma_{0}^{\prime}$. This implies that $S_{+}^{*}\left(S_{0}\right) \backslash \operatorname{Trap}\left(\Omega_{K}\right)$ is dense in $S_{+}^{*}\left(S_{0}\right)$.

Since $S_{+}^{*}\left(S_{0}\right)$ is a manifold and $\operatorname{Trap}\left(\Omega_{K}\right) \cap S_{+}^{*}\left(S_{0}\right)$ is compact, it follows from Proposition 2.4 that any two points in $S_{+}^{*}\left(S_{0}\right) \backslash \operatorname{Trap}\left(\Omega_{K}\right)$ can be connected by a $C^{1}$ curve lying entirely in $S_{+}^{*}\left(S_{0}\right) \backslash \operatorname{Trap}\left(\Omega_{K}\right)$. Denote by $S_{K}$ the set of the points $\sigma \in S_{+}^{*}\left(S_{0}\right) \backslash \operatorname{Trap}\left(\Omega_{K}\right)$ such that $\gamma_{K}(\sigma)$ is a simply reflecting ray. It follows from MS2 (cf. also Sect. 24.3 in $[\mathrm{H}]$ ) and Proposition 2.4 in [St2]) that $S_{K}$ is open and dense and has full Lebesgue measure in $S_{+}^{*}\left(S_{0}\right) \backslash \operatorname{Trap}\left(\Omega_{K}\right)$. Proposition 4.3 in [St4] shows that if $K, L$ have conjugate flows, then $S_{K}=S_{L}$. Moreover, using Proposition 6.3 in St4 and the above Proposition 2.4 we get the following.

Proposition 2.5. Let $K, L$ satisfy Hypothesis SCC. Then

$$
\#\left(\gamma_{K}(\sigma) \cap \partial K\right)=\#\left(\gamma_{L}(\sigma) \cap \partial L\right)
$$

for all $\sigma \in S_{K}=S_{L}$. 
Definition 2.6. A $C^{1}$ path $\sigma(s), 0 \leq s \leq a$ (for some $a>0$ ), in $S_{+}^{*}\left(S_{0}\right) \backslash \operatorname{Trap}\left(\Omega_{K}\right)$ will be called $K$-admissible if it has the following properties:

(i) $\sigma(0)$ generates a free ray in $\Omega_{K}$, i.e. a ray without any common points with $\partial K$

(ii) if $\sigma(s) \in M_{i}$ for some $i$ and $s \in[0, a]$, then $\sigma$ is transversal to $M_{i}$ at $\sigma(s)$ and $\sigma(s) \notin M_{j}$ for any submanifold $M_{j} \neq M_{i}$.

Thus, if a curve $\sigma(s), 0 \leq s \leq a$, in $S_{+}^{*}\left(S_{0}\right) \backslash \operatorname{Trap}\left(\Omega_{K}\right)$ is admissible, then the first point $\sigma(0)$ of this curve generates a free ray $\gamma_{K}^{+}(\sigma(0))$ in $\Omega_{K}$, i.e. a straight-line ray without common points with $K$. When $s$ moves gradually over the interval $[0, a]$ the ray $\gamma_{K}^{+}(\sigma(s))$ may start having common points with $\partial K$ and the number of common points may increase substantially 1 by the time $s$ becomes equal to $a$. Moreover, it follows from (ii) that if for some $s \in(0, a]$ the scattering ray $\gamma_{K}^{+}(\sigma(s))$ has a tangent point to $\partial K$, then it has only one tangent point to $\partial K$ and the tangency is of first order only. It is also clear from the above Definition that if the curve $\sigma(s)(0 \leq s \leq a)$ is admissible and $\rho(s)(0 \leq s \leq a)$ is uniformly close to $\sigma(s)$ (i.e. $\rho(s)$ and $\sigma(s)$ and their derivatives are $\epsilon$-close for all $s$ for some sufficiently small $\epsilon>0)$, then $\rho(s)$ is also admissible.

Notice that under Hypothesis SCC, we have $S_{+}^{*}\left(S_{0}\right) \backslash \operatorname{Trap}\left(\Omega_{K}\right)=S_{+}^{*}\left(S_{0}\right) \backslash$ $\operatorname{Trap}\left(\Omega_{L}\right)$ and a path is $K$-admissible iff it is $L$-admissible. So, in what follows we will just call such paths admissible.

Proposition 2.7. For any $\rho \in S_{+}^{*}\left(S_{0}\right) \backslash \operatorname{Trap}\left(\Omega_{K}\right)$ there exists an admissible path $\sigma(s), 0 \leq s \leq a$, with $\sigma(a)=\rho$.

Proof. This follows from Proposition 6.3 in [St4] (or rather its proof) and Propositions 2.3 and 2.4 above. We sketch the proof here for completeness.

Fix an arbitrary $\rho_{0} \in S_{+}^{*}\left(S_{0}\right) \backslash \operatorname{Trap}\left(\Omega_{K}\right)$ such that $\gamma_{K}^{+}\left(\rho_{0}\right)$ is a free ray in $\Omega_{K}$, i.e. it has no common points with $K$.

Let $\rho \in S_{+}^{*}\left(S_{0}\right) \backslash \operatorname{Trap}\left(\Omega_{K}\right)$. Since $S_{+}^{*}\left(S_{0}\right) \backslash \operatorname{Trap}\left(\Omega_{K}\right)$ is open in $S_{+}^{*}\left(S_{0}\right)$ and arc connected by Proposition 2.4, there exists a smooth curve $\sigma(s), 0 \leq s \leq 1$, in $S_{+}^{*}\left(S_{0}\right) \backslash \operatorname{Trap}\left(\Omega_{K}\right)$ such that $\sigma(0)=\rho_{0}$ and $\sigma(1)=\rho$. Now the compactness of $\operatorname{Trap}\left(\Omega_{K}\right) \cap S_{+}^{*}\left(S_{0}\right)$ implies the existence of a compact neighbourhood $W_{0}$ of $\gamma=\{\sigma(s): s \in[0,1]\}$ in $S_{+}^{*}\left(S_{0}\right)$ such that $W_{0} \cap \operatorname{Trap}\left(\Omega_{K}\right)=\emptyset$. Slightly perturbing the curve $\gamma$ in the interior of $W_{0}$, we may assume that $\gamma$ is transversal to any of the submanifolds $M_{i}$ and $R_{i}$ from Proposition 2.3 (cf. [Hi]). Since the codimension of $R_{i}$ in $S_{+}^{*}\left(S_{0}\right)$ is 2 , this implies $\gamma \cap R_{i}=\emptyset$ for each $i$. Moreover, $W_{0} \cap\left(\bigcup_{i} R_{i}\right)$ is compact, since $\left\{R_{i}\right\}$ is locally finite. Then shrinking $W_{0}$ we may assume that $W_{0} \cap R_{i}=\emptyset$ for all $i$. Now the fact that the family $\left\{M_{i}\right\}$ is locally finite in $S_{+}^{*}\left(S_{0}\right) \backslash \operatorname{Trap}\left(\Omega_{K}\right)$ implies that $\gamma \cap M_{i} \neq \emptyset$ only for finitely many $i$. So, there exist only finitely many $s_{1}, \ldots, s_{k} \in[0,1]$ such that $\gamma_{K}^{+}\left(\sigma\left(s_{j}\right)\right)$ contains a tangent point to $\partial K$, and moreover (since $\sigma\left(s_{j}\right) \notin R_{i}$ for any $i$ ), $\sigma\left(s_{j}\right)$ belongs to only one member of the family $\left\{M_{i}\right\}$ for all $j=1, \ldots, k$.

\section{Proof of Theorem 1.2}

Let $K$ and $L$ be as in Theorem 1.2. We will show that they coincide. A point $y \in \partial K$ will be called regular if $\partial K=\partial L$ in an open neighbourhood of $y$ in $\partial K$.

\footnotetext{
${ }^{1}$ Although in general this number is not a monotone function of $s$, so it may change in many different fashions.
} 
Otherwise $y$ will be called irregular. A point $y \in \partial K$ will be called accessible if there exists $\rho \in S_{+}^{*}\left(S_{0}\right) \backslash \operatorname{Trap}\left(\Omega_{K}\right)$ such that the trajectory $\gamma_{K}^{+}(\rho)$ contains the point $y$. Denote by $\mathcal{A}_{K}$ the set of all accessible points $y \in \partial K$.

Lemma 3.1. Let $d \geq 2$ and let $K$ have the form (1.1). Then $\mathcal{A}_{K}$ is dense in $\partial K$.

Proof. We will use a slight modification of an argument contained in the proof of Proposition 5.1 in [St2] (see also Proposition 2.4 above) which assumes $d \geq 3$. We will sketch this proof here dealing with the case $d=2$ as well.

First, it follows from Proposition 2.3(c) that there exists a countable locally finite family $\left\{Q_{i}\right\}$ of codimension 2 smooth submanifolds of $S_{\partial K}^{*}\left(\Omega_{K}\right)$ such that for any $\sigma \in S_{\partial K}^{*}\left(\Omega_{K}\right) \backslash\left(\bigcup_{i} Q_{i}\right)$ the trajectory $\gamma_{K}(\sigma)$ has at most one tangency to $\partial K$. Notice that when $d=2$, we have $\operatorname{dim}\left(S_{\partial K}^{*}\left(\Omega_{K}\right)\right)=2$, and then each $Q_{i}$ is a finite subset of $S_{\partial K}^{*}\left(\Omega_{K}\right)$, so $\bigcup_{i} Q_{i}$ is a discrete subset of $S_{\partial K}^{*}\left(\Omega_{K}\right)$.

Consider an arbitrary $x_{0} \in \partial K$ and let $\delta>0$. We will show that there exist points $x \in \mathcal{A}_{K}$ which are $\delta$-close to $x_{0}$. Take an arbitrary $u_{0} \in \mathbf{S}^{d-1}$ so that $\sigma_{0}=\left(x_{0}, u_{0}\right) \in S_{\partial K}^{*}\left(\Omega_{K}\right)$. Fix a small $\epsilon>0$ and set $X=\left\{x_{0}+\epsilon u:\left\|u-u_{0}\right\|<\epsilon\right\}$ and $\widetilde{X}=\left\{\left(x_{0}+\epsilon u, u\right):\left\|u-u_{0}\right\|\right\}$. As in the proof of Proposition 2.4, using Thom's Transversality Theorem (cf. e.g. [Hi]), and applying an arbitrarily small in the $C^{3}$ Whitney topology deformation to $X$, we get a $C^{3}$ convex surface $Y$ in $\Omega_{K}$ which is $C^{3}$-close to $X$ and so that $\tilde{Y}$ is transversal to each of the submanifolds $Q_{i}^{\prime}$. We take $Y$ so close to $X$ that for every $(y, v) \in \tilde{Y}$ for the point $x \in \partial K$ with $x+t v=y$ for some $t>0$ close to $\epsilon$ we have $\left\|x_{0}-x\right\|<\delta$. As in the proof of Proposition 2.4, when $d=2, \tilde{Y} \cap Q_{i}^{\prime}$ is a discrete subset of $\widetilde{Y}$, and when $d \geq 3$, for $Y^{\prime}=\tilde{Y} \cap\left(\bigcup_{i} Q_{i}\right)$ we have $\operatorname{dim}\left(Y^{\prime}\right) \leq d-3$.

Let $Y_{0}$ the set of those $\sigma \in \tilde{Y} \cap \operatorname{Trap}\left(\Omega_{K}\right)$ such that the trajectory $\gamma_{K}^{+}(\sigma)$ has no tangencies to $\partial K$, and let $\widetilde{F}=\prod_{r=0}^{\infty} F$, where $F=\left\{1,2, \ldots, k_{0}\right\}$. Fix an arbitrary $\theta \in(0,1)$ and consider the metric $d$ on $\widetilde{F}$ defined by $d\left(\left(x_{i}\right),\left(y_{i}\right)\right)=0$ if $x_{i}=y_{i}$ for all $i, d\left(\left(x_{i}\right),\left(y_{i}\right)\right)=1$ if $x_{0} \neq y_{0}$, and $d\left(\left(x_{i}\right),\left(y_{i}\right)\right)=\theta^{N}$ if $x_{i}=y_{i}$ for all $i=0,1, \ldots, N-1$ and $N \geq 1$ is maximal with this property. Then $\widetilde{F}$ is a compact totally disconnected metric space. Consider the map $f: Y_{0} \longrightarrow \widetilde{F}$, defined by $f(\sigma)=\left(i_{0}, i_{1}, \ldots\right)$, where the $j$ th reflection point of $\gamma_{K}^{+}(\sigma)$ belongs to $\partial K_{i_{j}}$ for all $j=0,1, \ldots$. Clearly, the map $f$ is continuous and, using the strict convexity of $Y$, it follows from St1 that $f^{-1}: f\left(Y_{0}\right) \longrightarrow Y_{0}$ is also continuous (it is in fact Lipschitz with respect to an appropriate metric on $\widetilde{F}$ ). Thus, $Y_{0}$ is homeomorphic to a closed subspace of $\widetilde{F}$ and therefore $Y_{0}$ is a compact totally disconnected subset of $\tilde{Y}$.

Given integers $p, q$ such that $q \in\left\{1, \ldots, k_{0}\right\}$ and $p \geq 1$, denote by $Y(p, q)$ the set of those $\sigma \in \widetilde{Y} \cap \operatorname{Trap}\left(\Omega_{K}\right)$ such that $\gamma_{K}^{+}(\sigma)$ has exactly one tangency to $\partial K$ which is its $p$ th reflection point and it belongs to $\partial K_{q}$. Given $p, q$, define $f: Y(p, q) \longrightarrow \widetilde{F}$ as above and notice that in the present case we have $i_{q}=p$ for any $\sigma \in Y(p, q)$, where $f(\sigma)=\left(i_{j}\right)$. This and the definition of $Y(p, q)$ imply that $f$ is continuous on $Y(p, q)$ and using [St1] again, we get that $Y(p, q)$ is homeomorphic to a closed subspace of $\widetilde{F}$ and so $Y(p, q)$ is a compact totally disconnected subset of $S_{\partial K}^{*}\left(\Omega_{K}\right)$.

Thus, each of the sets $Y^{\prime}, Y_{0}$ and $Y(p, q)$ is a subset of first Baire category in $\widetilde{Y}$. It now follows from Baire's category theorem that $Y^{\prime} \cup Y_{0} \cup \bigcup_{p, q} Y(p, q)$ is nowhere dense in $\tilde{Y}$. Thus, $\mathcal{A}_{K} \cap \tilde{Y} \supseteq \tilde{Y} \backslash\left(Y^{\prime} \cup Y_{0} \cup \bigcup_{p, q} Y(p, q)\right)$ is dense in $\tilde{Y}$. 
Thus, there exists $(y, v) \in \widetilde{Y}$ which generates a non-trapped trajectory. By the choice of $Y$, if $x \in \partial K$ is so that $x+t v=y$ for some $t>0$ close to $\epsilon$, then we have $\left\|x_{0}-x\right\|<\delta$. Clearly, $x \in \mathcal{A}_{K}$, so $x_{0}$ is $\delta$-close to a point in $\mathcal{A}_{K}$. This proves the assertion.

Definition 3.2. For any integer $n \geq 1$ let $Z_{n}$ be the set of those irregular points $x \in$ $\partial K$ for which there exists an admissible path $\sigma(s), 0 \leq s \leq a$, in $S_{+}^{*}\left(S_{0}\right) \backslash \operatorname{Trap}\left(\Omega_{K}\right)$ such that $\sigma(0)$ generates a free ray in $\mathbb{R}^{d}, x$ belongs to the billiard trajectory $\gamma_{K}^{+}(\sigma(a))$, and for any $s \in[0, a]$ the trajectory $\gamma_{K}^{+}(\sigma(s))$ has at most $n$ irregular common points with $\partial K$.

Notice that in the above definition, the billiard trajectory $\gamma_{K}^{+}(\sigma(s))$ may have more than $n$ common points with $\partial K$ - at most $n$ of them will be irregular and all the others must be regular. We will prove by induction on $n$ that $Z_{n}=\emptyset$ for all $n \geq 1$.

First, we will show that $Z_{1}=\emptyset$. Let $x \in Z_{1}$. Then there exists a $C^{1}$ path $\sigma(s)$, $0 \leq s \leq a$, in $S_{+}^{*}\left(S_{0}\right)$ as in the definition of $Z_{1}$. In particular, $x$ lies on $\gamma_{K}^{+}(\sigma(a))$ and for each $s \in[0, a]$ the trajectory $\gamma_{K}^{+}(\sigma(s))$ has at most 1 irregular point. As in the argument below dealing with the inductive step, we may assume that $a>0$ is the smallest number for which $\gamma_{K}^{+}(\sigma(a))$ has an irregular point, i.e. for all $s \in[0, a)$ the trajectory $\gamma_{K}^{+}(\sigma(s))$ contains no irregular points at all. Set $\rho=\sigma(a)$ and $\gamma=\gamma_{K}^{+}(\sigma(a))$.

Before continuing let us remark that $\gamma=\gamma_{K}^{+}(\rho)$ and $\gamma^{\prime}=\gamma_{L}^{+}(\rho)$ must have the same number of common points with $\partial K$ and $\partial L$, respectively. Indeed, if $\gamma$ has a tangent point to $\partial K$ (then $\rho \in M_{i}$ for some, unique, $i$ ), then $\gamma$ has only one tangent point to $\partial K$ and $\gamma^{\prime}$ also has a unique tangent point to $\partial L$. Let $k$ be the number of proper reflection points of $\gamma$ at $\partial K$. Then we can find $\rho^{\prime} \in S_{+}^{*}\left(S_{0}\right) \backslash \operatorname{Trap}\left(\Omega_{K}\right)$ arbitrarily close to $\rho$ so that $\gamma_{K}^{+}\left(\rho^{\prime}\right)$ has exactly $k$ common points with $\partial K$, all of them being proper reflection points. By Proposition 2.5, $\gamma_{L}^{+}\left(\rho^{\prime}\right)$ also has exactly $k$ common points with $\partial L$, all of them being proper reflection points. Thus, $\gamma_{L}^{+}\left(\rho^{\prime}\right)$ also has at most $k$ proper reflection points, so

$\sharp$ (proper reflection points of $\left.\gamma_{K}^{+}(\rho)\right) \geq \sharp$ (proper reflection points of $\gamma_{L}^{+}(\rho)$ ).

By symmetry, it follows that $\gamma_{K}^{+}(\rho)$ and $\gamma_{L}^{+}(\rho)$ have the same number of proper reflection points, and therefore the same number of common points with $\partial K$ and $\partial L$, respectively.

Let $x_{1}, \ldots, x_{m}$ be the common points of $\gamma_{K}^{+}(\rho)$ and $\partial K$ and let $x_{i}=x$ for some $i$. Then for each $j \neq i$ there exists an open subset $U_{j}$ of $\partial K$ such that $U_{j}=U_{j} \cap \partial L$. Let $\rho=\left(x_{0}, u_{0}\right)$ and let $\omega=\left(x_{m+1}, u_{m+1}\right) \in S^{*}\left(S_{0}\right)$ be the last common point of $\gamma_{K}^{+}(\rho)$ with $S^{*}\left(S_{0}\right)$ before it goes to $\infty$. Let $\mathcal{F}_{t_{j}}^{(K)}(\rho)=\left(x_{j}, u_{j}\right), 1 \leq j \leq m+1$. It then follows from the above that $\mathcal{F}_{t}^{(K)}(\rho)=\mathcal{F}_{t}^{(L)}(\rho)$ for $0 \leq t<t_{i}$ and also, $\mathcal{F}_{\tau}^{(K)}(\omega)=\mathcal{F}_{\tau}^{(L)}(\omega)$ for all $-\left(t_{m+1}-t_{i}\right)<\tau \leq 0$. So, the trajectories $\gamma_{K}^{+}(\rho)$ and $\gamma_{L}^{+}(\rho)$ both pass through $x_{i-1}$ with the same (reflected) direction $u_{i-1}$ and through $x_{i+1}$ with the same (reflected) direction $u_{i+1}$. Thus, $x_{1}, \ldots, x_{i-1}, x_{i+1}, \ldots, x_{m}$ are common points of $\gamma_{L}^{+}(\rho)$ and $\partial L$. As observed above, $\gamma_{L}^{+}(\rho)$ must have exactly $m$

\footnotetext{
${ }^{2}$ We should probably remark that $\gamma$ may have more than one common point with $\partial K$, and for some $s<a, \gamma_{K}^{+}(\sigma(s))$ may have common points with $\partial K$, however all of them will be regular points.
} 
common points with $\partial L$, so it has a common point $y_{i}$ with $\partial L$ 'between' $x_{i-1}$ and $x_{i+1}$.

Next, we consider two cases.

Case 1. $x_{i}$ is a proper reflection point of $\gamma$ at $\partial K$. It then follows immediately from the above that $x_{i}$ lies on $\gamma^{\prime}$ and $\gamma^{\prime}$ has a proper reflection point at $x_{i}$, so in particular $x_{i} \in \partial L$. Moreover for any $y \in \partial K$ sufficiently close to $x_{i}$ there exists $\rho^{\prime} \in S_{+}^{*}\left(S_{0}\right) \backslash \operatorname{Trap}\left(\Omega_{K}\right)$ close to $\rho$ so that $\gamma_{K}^{+}\left(\rho^{\prime}\right)$ has a proper reflection point at $y$. Then repeating the above argument and using again $U_{j}=U_{j} \cap \partial L$ for $j \neq i$, shows that $y \in \partial L$. Thus, $\partial K=\partial L$ in an open neighbourhood of $x=x_{i}$ in $\partial K$, which is a contradiction with the assumption that $x$ is an irregular point.

Case 2. $x_{i}$ is a tangent point of $\gamma$ to $\partial K$. Then $\gamma$ (and so $\gamma^{\prime}$ ) has $m-1$ proper reflection points and $y_{i}$ is a point on the segment $\left[x_{i-1}, x_{i+1}\right]$. Assume for a moment that $y_{i} \neq x_{i}$. Clearly we can choose $x_{i}^{\prime} \in \partial K$ arbitrarily close to $x_{i}$ and $u_{i}^{\prime} \in \mathbf{S}^{d-1}$ close to $u_{i}$ so that $u_{i}^{\prime}$ is tangent to $\partial K$ at $x_{i}^{\prime}$ and the straight line determined by $x_{i}^{\prime}$ and $u_{i}^{\prime}$ intersects $\partial L$ transversally near $y_{i}$. Let $\rho^{\prime} \in S_{+}^{*}\left(S_{0}\right) \backslash \operatorname{Trap}\left(\Omega_{K}\right)$ be the point close to $\rho$ which determines a trajectory $\gamma_{K}^{+}\left(\rho^{\prime}\right)$ passing through $x_{i}^{\prime}$ in direction $u_{i}^{\prime}$, i.e. tangent to $\partial K$ at $x_{i}^{\prime}$. Then $\gamma_{K}^{+}\left(\rho^{\prime}\right)$ has $m-1$ proper reflection points and one tangent point, while $\gamma_{L}^{+}\left(\rho^{\prime}\right)$ has $m$ proper reflection points and no tangent points at all. This is impossible, so we must have $y_{i}=x_{i}$. Moreover, using a similar argument one shows that every $x^{\prime} \in \partial K$ sufficiently close to $x_{i}$ belongs to $\partial L$, as well. So, $x_{i}$ is a regular point, contradicting our assumption.

This proves that $Z_{1}=\emptyset$. Next, suppose that for some $n>1$ we have $Z_{1}=$ $\ldots=Z_{n-1}=\emptyset$ and that $Z_{n} \neq \emptyset$. Let $x \in Z_{n}$. Then there exists an admissible $C^{1}$ path $\sigma(s), 0 \leq s \leq a$, in $S_{+}^{*}\left(S_{0}\right)$ as in the definition of $Z_{n}$. In particular, $x$ lies on $\gamma_{K}^{+}(\sigma(a))$ and for each $s \in[0, a]$ the trajectory $\gamma_{K}(\sigma(s))$ has at most $n$ irregular points. Set

$$
A=\left\{s \in(0, a]: \gamma_{K}^{+}(\sigma(s)) \text { has } n \text { irregular points }\right\} .
$$

Clearly $A \neq \emptyset$, since by assumption $x \in Z_{n}$ and $Z_{j}=\emptyset$ for $j<n$. Set $b=\inf A$. Then there exists a decreasing sequence $\left\{s_{m}\right\} \subset(0, a]$ with $s_{m} \searrow b$ and such that for each $m \geq 1$ the trajectory $\gamma_{K}^{+}\left(\sigma\left(s_{m}\right)\right)$ contains $n$ distinct irregular (consecutive) points $x_{1}^{(m)}, \ldots, x_{n}^{(m)}$. Since $K$ has the form (1.1), these points belong to distinct connected components of $K$. Choosing an appropriate subsequence of $\left\{s_{m}\right\}$, we may assume that $x_{i}^{(m)}$ belongs to the same connected component $K_{j_{i}}$ for all $m \geq 1$ and there exists $x_{i}=\lim _{m \rightarrow \infty} x_{i}^{(m)}$ for all $i=1, \ldots, n$. Then $x_{1}, \ldots, x_{n}$ are distinct common points of $\gamma_{K}^{+}(\sigma(b))$ with $\partial K$. Moreover, if some $x_{i}$ is a regular point, then $\partial K=\partial L$ in an open neighbourhood of $x_{i}$ in $\partial K$, so $x_{i}^{(m)}$ would be regular for large $m$, a contradiction. Thus, all $x_{1}, \ldots, x_{n}$ are irregular points.

The above reasoning shows that we may assume $b=a, \gamma_{K}^{+}((a))$ contains exactly $n$ irregular points, and for any $s \in[0, a)$ the trajectory $\gamma_{K}^{+}(\sigma(s))$ has $<n$ irregular points. Now the inductive assumption implies that for any $s \in[0, a)$ the trajectory $\gamma_{K}^{+}(\sigma(s))$ contains no irregular points at all. Since $Z_{j}=\emptyset$ for $j<n$, every irregular point of $\gamma_{K}^{+}(\sigma(a))$ must belong to $Z_{n}$.

Set $\gamma=\gamma_{K}^{+}(\sigma(a))$ for brevity. Let $x_{1}, \ldots, x_{n}$ be the consecutive irregular points of $\gamma$ (which may have some other common points with $\partial K$ ) and for $s<a$ close to $a$, let $x_{1}(s), \ldots, x_{n}(s)$ be the consecutive common points of $\gamma_{K}^{+}(\sigma(s))$ with $\partial K$ such that $x_{i}(s)$ lies on the connected component $K_{j_{i}}$ of $K$ containing $x_{i}(i=1, \ldots, n)$. 
Then for every $i, \partial K=\partial L$ in an open neighbourhood of $x_{i}(s)$ in $\partial K$ for $s<a$ close to $a$, so there exists an open subset $U_{i}$ of $\partial K$ with $x_{i} \in \overline{U_{i}}$ and $\partial K \cap U_{i}=\partial L \cap U_{i}$. Next, we consider two cases.

Case 1. $\gamma$ contains no tangent points to $\partial K$.

Choose a small $\delta>0$ (how small will be determined later). We will replace the path $\sigma(s)$ by another one $\tilde{\sigma}(s), 0 \leq s \leq a$, such that $\tilde{\sigma}(s)=\sigma(s)$ for $s \in[0, a-2 \delta]$. Let $\mathcal{F}_{t_{1}}^{(K)}\left(x_{1}, u_{1}\right)=\left(x_{2}, u_{2}\right)$ for some $t_{1}>0$, where $u_{1} \in \mathbf{S}^{d-1}$ is the (reflected) direction of $\gamma$ at $x_{1}$ and $u_{2} \in \mathbf{S}^{d-1}$ is the reflected direction of $\gamma$ at $x_{2}$. Take a small $\epsilon>0$ and set

$$
X:=\left\{x_{1}+\epsilon u: u \in \mathbf{S}^{d-1},\left\|u-u_{1}\right\|<\delta\right\}
$$

and

$$
\widetilde{X}:=\left\{\left(x_{1}+\epsilon u, u\right): u \in \mathbf{S}^{d-1},\left\|u-u_{1}\right\|<\delta\right\} .
$$

Let $u_{1}(s)$ be the (reflected) direction of the trajectory $\sigma(s)$ at $x_{1}(s)$. Take $t^{\prime}<t_{1}$ close to $t_{1}$ and set $\widetilde{Y}=\mathcal{F}_{t^{\prime}}^{(K)}(\widetilde{X}), Y=\operatorname{pr}_{1}(\widetilde{Y})$. Then

$$
\widetilde{Y}=\left\{\left(y, \nu_{Y}(y)\right): y \in Y\right\}
$$

where $\nu_{Y}(y)$ is the unit normal to $Y$ at $y$ in the direction of the flow $\mathcal{F}_{t}^{(K)}$, and $\left(y_{1}, v_{1}\right)=\mathcal{F}_{t^{\prime}}^{(K)}\left(x_{1}, u_{1}\right) \in \tilde{Y}$, so $y_{1} \in Y$.

Set

$$
\widehat{Y}=\left\{(y, v): y \in Y, v \in \mathbf{S}^{d-1},\left\|v-\nu_{Y}(y)\right\|<\epsilon\right\} .
$$

There exists an open neighbourhood $V$ of $\left(x_{1}, u_{1}\right)$ in $S_{\partial K}^{*}\left(\Omega_{K}\right)$ such that the shift $\Phi: V \longrightarrow \widehat{Y}$ along the flow $\mathcal{F}_{t}^{(K)}$ is well-defined and smooth. Assuming $\delta$ is sufficiently small, $\left(y_{1}(s), v_{1}(s)\right)=\Phi\left(x_{1}(s), u_{1}(s)\right)$ is well-defined for $s \in[a-2 \delta, a]$. Then $y_{1}(s), s \in[a-2 \delta, a]$, is a $C^{1}$ curve on $Y$ and the ray issued from $y_{1}(s)$ in direction $v_{1}(s)$ hits $\partial K_{j_{2}}$ at $x_{2}(s)$. Moreover, $v_{1}(a)=\nu_{Y}\left(y_{1}\right)$.

Assuming $\delta>0$ is sufficiently small, for all $s, s^{\prime} \in[a-2 \delta, a]$, there exists a unique vector $v_{1}\left(s, s^{\prime}\right) \in \mathbf{S}^{d-1}$ such that

$$
y_{1}(s)+t\left(s, s^{\prime}\right) v_{1}\left(s, s^{\prime}\right)=x_{2}\left(s^{\prime}\right)
$$

for some $t\left(s, s^{\prime}\right)$ close to $t_{1}-t^{\prime}$. Moreover, $v_{1}\left(s, s^{\prime}\right)$ and $t\left(s, s^{\prime}\right)$ are smoothly $\left(C^{1}\right)$ depending on $s$ and $s^{\prime}$. Clearly, $v_{1}(s, s)=v_{1}(s)$.

Since $Y$ is a strictly convex surface with a unit normal field $\nu_{Y}(y)$, it is clear that for any $s \in(0, a]$ sufficiently close to $a$ there exists $y \in Y$ close to $y_{1}$ such that $y+t \nu_{Y}(y)=x_{2}(s)$ for some $t$ close to $t_{1}-t^{\prime}$. Fix a small $\delta>0$, set $s_{0}=a-\delta / 2$ and let $\tilde{y} \in Y$ be so that

$$
\tilde{y}+t \nu_{Y}(\tilde{y})=x_{2}\left(s_{0}\right) \in U_{2}
$$

for some $t>0$ close to $t_{1}-t^{\prime}$. Take a $C^{1}$ curve $\tilde{y}_{1}(s), s \in[a-2 \delta, a]$, such that $\tilde{y}_{1}(s)=y_{1}(s)$ for $s \in[a-2 \delta, a-3 \delta / 2]$ and $\tilde{y}_{1}(a)=\tilde{y}$.

Next, define $\hat{s}(s), s \in[0, a]$, by $\hat{s}(s)=s$ for $0 \leq s \leq a-\delta$ and

$$
\hat{s}(s)=\frac{s}{2}+s_{0}-\frac{a}{2}=s_{0}-\frac{a-s}{2} \in\left[a-\delta, s_{0}\right], \quad s \in[a-\delta, a] .
$$

Then $\hat{s}$ is continuous however not differentiable at $s=a-\delta$. Take a $C^{1}$ function $\tilde{s}(s), s \in[0, a]$, which coincides with $\hat{s}$ on $\left[0, a-\delta-\delta_{1}\right]$ and on $\left[a-\delta+\delta_{1}, a\right]$ for 
some $\delta_{1}<\delta / 2$ so that the range of $\tilde{s}$ is the same as that of $\hat{s}$, i.e. it coincides with the interval $\left[0, s_{0}\right]$. Now for any $s \in[a-2 \delta, a]$ take $\tilde{v}_{1}(s)$ so that

$$
\tilde{y}_{1}(s)+\tilde{t}(s) \tilde{v}_{1}(s)=x_{2}(\tilde{s}(s)), \quad s \in[a-2 \delta, a],
$$

for some $\tilde{t}(s)$ close to $t_{1}-t^{\prime}$. For $s \in\left[a-2 \delta, a-\delta-\delta_{1}\right]$ we have $\tilde{y}_{1}(s)=y_{1}(s)$ and $\tilde{s}(s)=s$, which imply $\tilde{v}_{1}(s)=v_{1}(s)$. When $s=a$ we have $\tilde{y}_{1}(a)=\tilde{y}$ and $\tilde{s}(a)=\hat{s}(a)=s_{0}$, so by $(3.1)$ we must have $\tilde{v}_{1}(a)=\nu_{Y}(\tilde{y})$, i.e. $\left(\tilde{y}_{1}(a), \tilde{v}_{1}(a)\right) \in \tilde{Y}$, and therefore

$$
\operatorname{pr}_{1}\left(\Phi^{-1}\left(\tilde{y}_{1}(a), \tilde{v}_{1}(a)\right)\right)=\operatorname{pr}_{1}\left(\mathcal{F}_{-t^{\prime}}^{(K)}\left(\tilde{y}_{1}(a), \tilde{v}_{1}(a)\right)\right)=x_{1} .
$$

Set $\left(\tilde{x}_{1}(s), \tilde{u}_{1}(s)\right)=\Phi^{-1}\left(\tilde{y}_{1}(s), \tilde{v}_{1}(s)\right), s \in[a-2 \delta, a]$. For $s \in\left[a-2 \delta, a-\delta-\delta_{1}\right]$, we have $\tilde{s}(s)=\hat{s}(s)=s$ and therefore $\tilde{y}_{1}(s)=y_{1}(s)$ and $\tilde{v}_{1}(s)=v_{1}(s)$, which gives $\left(\tilde{x}_{1}(s), \tilde{u}_{1}(s)\right)=\left(x_{1}(s), u_{1}(s)\right)$.

Now define the path $\tilde{\sigma}(s), 0 \leq s \leq a$, on $S_{+}^{*}\left(S_{0}\right)$ by $\tilde{\sigma}(s)=\sigma(s)$ for $s \in$ $[0, a-2 \delta]$, and for $s \in[a-2 \delta, a]$ let $\tilde{\sigma}(s)$ be the unique point such that $\mathcal{F}_{\tilde{t}_{0}(s)}^{(K)}(\tilde{\sigma}(s))=$ $\left(\tilde{x}_{1}(s), \tilde{u}_{1}(s)\right)$ for some $\tilde{t}_{0}(s)$ close to $t_{0}(s)$, where $\mathcal{F}_{t_{0}(s)}^{(K)}(\sigma(s))=\left(x_{1}(s), u_{1}(s)\right)$. It follows from (3.2) that $\tilde{\sigma}(a)$ contains the point $x_{1}$. Moreover the construction of $\tilde{\sigma}$ shows that it is a $C^{1}$ path. Assuming $\delta$ is sufficiently small, $\tilde{\sigma}(s)$ has no tangent points to $\partial K$ for all $s \in[a-2 \delta, a]$, so $\tilde{\sigma}(s)$ is an admissible path. For $s \in[0, a-2 \delta]$, $\gamma_{K}(\tilde{\sigma}(s))=\gamma_{K}(\sigma(s))$ contains no irregular points. For $s \in[a-2 \delta, a], \gamma_{K}(\tilde{\sigma}(s))$ has at most $n$ irregular points, close to $x_{1}, x_{2}, \ldots, x_{n}$ (if any). If for some $s \in[a-2 \delta, a]$, $\gamma_{K}(\tilde{\sigma}(s))$ has exactly $n$ irregular points, then the second of these must be $x_{2}(\tilde{s}(s))$. However, $\tilde{s}(s)<a$ for all such $s$, so $x_{2}(\tilde{s}(s)) \in U_{2}$, and therefore that cannot be the case. Thus, for all $s \in[a-2 \delta, a], \gamma_{K}(\tilde{\sigma}(s))$ has not more than $n-1$ irregular points, and therefore $x_{1}$ (and every other irregular point on $\gamma_{K}(\tilde{\sigma}(a))$ ) belongs to $Z_{n-1}$. This is a contradiction with the inductive assumption that $Z_{j}=\emptyset$ for all $j=1, \ldots, n-1$.

Case 2. $\gamma$ contains a tangent point $y_{0}$ to $\partial K$ (this may be one of the irregular points $x_{i}$ ).

Let $y_{0} \in \partial K_{p}$ for some $p$. According to the definition of an admissible path, $\gamma$ has only one tangent point to $\partial K$, so all other common points are proper reflection points. Since $n>1$, at least one of the irregular points on $\gamma$ is a proper reflection point, and at least one of them is different from $y_{0}$. We will assume $x_{1} \neq y_{0}$; the general case is very similar. As in Case 1, we will replace the path $\sigma(s)$ by another one $\tilde{\sigma}(s), 0 \leq s \leq a$, such that $\tilde{\sigma}(s)=\sigma(s)$ for $s \in[0, a-2 \delta]$ for some small $\delta>0$.

Let $\mathcal{F}_{\tau}^{(K)}\left(x_{1}, u_{1}\right)=\left(y_{0}, v_{0}\right)$ for some $\tau \in \mathbb{R}$ (which may be positive or negative), where $u_{1} \in \mathbf{S}^{d-1}$ is the (reflected) direction of $\gamma$ at $x_{1}$ and $v_{0}$ is the direction of $\gamma$ at $y_{0}$. Take a small $\epsilon>0$ and let

$$
X:=\left\{x_{1}+\epsilon u: u \in \mathbf{S}^{d-1},\left\|u-u_{1}\right\|<\delta\right\}
$$

and

$$
\widetilde{X}:=\left\{\left(x_{1}+\epsilon u, u\right): u \in \mathbf{S}^{d-1},\left\|u-u_{1}\right\|<\delta\right\} .
$$

Assuming $\delta>0$ is small enough, for $s \in[a-2 \delta, a]$ the trajectory $\gamma_{K}(\sigma(s))$ has a proper reflection point $x_{1}(s) \in \partial K_{j_{1}}$. Let $u_{1}(s)$ be the (reflected) direction of the trajectory $\sigma(s)$ at $x_{1}(s)$.

Clearly in the present case we have $\sigma(a) \in M_{i}$ for some $i$. By the definition of an admissible path, $\sigma(a) \notin M_{j}$ for any $j \neq i$ and moreover $\sigma$ is transversal 
to $M_{i}$ at $\sigma(a)$. Let $\sigma(s)=\left(x_{0}(s), u_{0}(s)\right) \in S_{+}^{*}\left(S_{0}\right)$ and let $\mathcal{F}_{t_{0}(s)}^{(K)}\left(x_{0}(s), u_{0}(s)\right)=$ $\left(x_{1}(s), u_{1}(s)\right)$. The shift $\Phi: S_{+}^{*}\left(S_{0}\right) \longrightarrow S_{\partial K}^{*}\left(\Omega_{K}\right)$ along the flow $\mathcal{F}_{t}^{(K)}$ is welldefined on an open neighbourhood $V_{0}$ of $\left(x_{0}, u_{0}\right)=\left(x_{0}(a), u_{0}(a)\right)$ and defines a diffeomorphism $\Phi: V_{0} \longrightarrow V=\Phi\left(V_{0}\right)$ for some small open neighbourhood $V$ of $\left(x_{1}, u_{1}\right)$ in $S_{\partial K}^{*}\left(\Omega_{K}\right)$. Since $M_{i}$ is a submanifold of $S_{+}^{*}\left(S_{0}\right)$ of codimension one, we can take $V_{0}$ so that $V_{0} \backslash M_{i}$ has two (open) connected components (separated by $M_{i}$ ) - each of them diffeomorphic to an open half-ball. We take $V_{0}$ so small that $V_{0} \cap M_{j}=\emptyset$ for all $j \neq i$.

Setting $M_{i}^{\prime}=\Phi\left(M_{i} \cap V_{0}\right)$, we get a similar picture in $V$, namely $V \backslash M_{i}^{\prime}$ has two (open) connected components (separated by $M_{i}^{\prime}$ ) - each of them diffeomorphic to an open half-ball. Assuming $\delta$ is sufficiently small, we have $\sigma(s) \in V_{0}$ for all $s \in[a-2 \delta, a]$ and $\sigma$ is transversal to $M_{i}$ at $\sigma(a)=\left(x_{0}, u_{0}\right)$. Thus, the curve $\left(x_{1}(s), u_{1}(s)\right), s \in[a-2 \delta, a]$, in $V$ is transversal to $M_{i}^{\prime}$ at $\left(x_{1}, u_{1}\right)$, so it must be contained in one of the connected components of $V \backslash M_{i}^{\prime}$. Denote by $V_{+}$the connected component of $V \backslash M_{i}^{\prime}$ that contains $\left(x_{1}(s), u_{1}(s)\right)$ for $s \in[a-2 \delta, a)$.

Finally, to get this picture near the tangent point $y_{0}$, take $\tau^{\prime} \in(\tau-\delta, \tau-\delta / 2)$ and set

$$
F=\left\{\left(x_{1}, u\right): u \in \mathbf{S}^{d-1},\left\|u-u_{1}\right\|<\delta\right\} \subset V, \quad \tilde{Y}=\mathcal{F}_{\tau^{\prime}}^{(K)}(F),
$$

and $Y=\operatorname{pr}_{1}(\tilde{Y})$. Then $\tilde{Y}=\left\{\left(y, \nu_{Y}(y)\right): y \in Y\right\}$, where $\nu_{Y}(y)$ is the unit normal to $Y$ at $y$ in the direction of the flow $\mathcal{F}_{t}^{(K)}$, and $\left(y_{1}, v_{1}\right)=\mathcal{F}_{\tau^{\prime}}^{(K)}\left(x_{1}, u_{1}\right) \in \tilde{Y}$, so $y_{1} \in Y$. Let $\Psi: F \longrightarrow \tilde{Y}$ be the shift along the flow $\mathcal{F}_{t}^{(K)}$. Assuming that $\delta$ is sufficiently small, this defines a diffeomorphism $\Psi: F \longrightarrow G$ between $F$ and an open subset $G$ of $\widetilde{Y}$. Set $M_{i}^{\prime \prime}=\Psi\left(F \cap M_{i}^{\prime}\right)$. Then $M_{i}^{\prime \prime}$ consists of those $\left(y, \nu_{Y}(y)\right) \in G$ that generate trajectories tangent to $\partial K$ (and this can only happen in the vicinity of $y_{0}$ on $\partial K_{p}$ ). It is clear (by a direct observation using the convexity of $K_{p}$ ) that $G \backslash M_{i}^{\prime \prime}$ has two connected components. Let $G_{+}$be the one with $\Psi^{-1}\left(G_{+}\right) \subset V_{+}$. Thus, there exists $v_{1}^{\prime} \in \mathbf{S}^{d-1},\left\|v_{1}^{\prime}-v_{1}\right\|<\delta$ such that $\left(y_{1}, v_{1}^{\prime}\right) \in G_{+}$. Applying $\Psi^{-1}$, this gives $u_{1}^{\prime} \in \mathbf{S}^{d-1}$ with $\left\|u_{1}^{\prime}-u_{1}\right\|<\delta$ such that $\left(x_{1}, u_{1}^{\prime}\right) \in V_{+}$.

Since $V_{+}$is connected (in fact diffeomorphic to an open half-ball), there exists a $C^{1}$ curve $\left(\tilde{x}_{1}(s), \tilde{u}_{1}(s)\right), s \in[a-2 \delta, a]$, in $V_{+}$such that $\left(\tilde{x}_{1}(s), \tilde{u}_{1}(s)\right)=\left(x_{1}(s), u_{1}(s)\right)$ for $s \in[a-2 \delta, a-\delta]$ and $\left(\tilde{x}_{1}(a), \tilde{u}_{1}(a)\right)=\left(x_{1}, u_{1}^{\prime}\right)$. Define the path $\tilde{\sigma}(s)$ by $\tilde{\sigma}(s)=\sigma(s)$ for $s \in[0, a-2 \delta]$ and $\tilde{\sigma}(s)=\Phi^{-1}\left(\tilde{x}_{1}(s), \tilde{u}_{1}(s)\right)$ for $s \in[a-2 \delta, a]$. It is clear from the construction that $\tilde{\sigma}$ is a $C^{1}$ path in $S_{+}^{*}\left(S_{0}\right)$. From the properties of $\sigma$, we have that for $s \in[0, a-2 \delta]$ the trajectory $\gamma_{K}(\tilde{\sigma}(s))$ does not contain any irregular points. The choice $V_{0}$ and that of the curve $\left(\tilde{x}_{i}(s), \tilde{u}_{1}(s)\right), s \in[a-2 \delta, a]$, show that $\gamma_{K}(\tilde{\sigma}(s))$ has no tangencies to $\partial K$ for any $s \in[a-2 \delta, a]$. On the other hand, $\gamma_{K}(\tilde{\sigma}(a))$ contains the irregular point $x_{1}$ (and therefore must have $n$ irregular points, since $Z_{j}=\emptyset$ for $\left.j<n\right)$. Now repeating the argument from Case 1 we get a contradiction. Thus, we must have $Z_{n}=\emptyset$.

This completes the induction process and proves that $Z_{n}=\emptyset$ for all $n \geq 1$.

We will now prove that $\partial K \subseteq \partial L$. By Lemma 3.1, $\mathcal{A}_{K}$ is dense in $\partial K$, so it is enough to show that $\mathcal{A}_{K} \subseteq \partial L$. Given $x \in \mathcal{A}_{K}$, by the definition of $\mathcal{A}_{K}$ (see the beginning of Sect. 3), $x$ is accessible, i.e. there exists $\rho \in S_{+}^{*}\left(S_{0}\right) \backslash \operatorname{Trap}\left(\Omega_{K}\right)$ such that $\gamma_{K}^{+}(\rho)$ has a reflection point at $x$. Then by Propositions 2.4 and 2.7 , there exists an admissible path $\sigma(s), 0 \leq s \leq a$, for some $a>0$, with $\rho^{\prime}=\sigma(a)$ arbitrarily close to $\rho$. Then $\gamma_{K}^{+}\left(\sigma\left(\rho^{\prime}\right)\right)$ has a reflection point $x^{\prime}$ at $\partial K$ near $x$. Since there are 
no irregular points on $\sigma(a)$, it follows that $\partial K=\partial L$ on an open neighbourhood of $x^{\prime}$. Thus, $x$ is arbitrarily close to $\partial L$, so we must have $x \in \partial L$. This proves that $\partial K \subseteq \partial L$.

By symmetry, $\partial L \subseteq \partial K$, so we have $\partial K=\partial L$.

\section{ACKNOWLEDGEMENT}

Thanks are due to the referee for a number of useful remarks and suggestions.

\section{REFERENCES}

[F] V. V. Fedorchuk, The fundamentals of dimension theory, In: General Topology I, A.V. Arhagel'skii and L. S. Pontryagin (Eds.), Encyclopedia of Mathematical Sciences Vol. 17, Springer-Verlag, 1990.

[G] V. Guillemin, Sojourn times and asymptotic properties of the scattering matrix, Proceedings of the Oji Seminar on Algebraic Analysis and the RIMS Symposium on Algebraic Analysis (Kyoto Univ., Kyoto, 1976), Publ. Res. Inst. Math. Sci. 12 (1976/77 supplement), 69-88. MR0448453 (56 \#6759)

[Hi] Morris W. Hirsch, Differential topology, Springer-Verlag, New York-Heidelberg, 1976. Graduate Texts in Mathematics, No. 33. MR0448362 (56 \#6669)

$[\mathrm{H}] \quad$ L. Hörmander, The analysis of linear partial differential operators, vol. III. Berlin: Springer, 1985.

[LP1] Peter D. Lax and Ralph S. Phillips, Scattering theory, Pure and Applied Mathematics, Vol. 26, Academic Press, New York-London, 1967. MR0217440 (36 \#530)

[LP2] Peter D. Lax and Ralph S. Phillips, The scattering of sound waves by an obstacle, Comm. Pure Appl. Math. 30 (1977), no. 2, 195-233. MR0442510 (56 \#892)

[Ma] Andrew Majda, A representation formula for the scattering operator and the inverse problem for arbitrary bodies, Comm. Pure Appl. Math. 30 (1977), no. 2, 165-194. MR0435625 (55 \#8583)

[MaR] Andrew Majda and James Ralston, An analogue of Weyl's theorem for unbounded domains. I, Duke Math. J. 45 (1978), no. 1, 183-196. MR0481639 (58 \#1744)

[M] Richard B. Melrose, Geometric scattering theory, Stanford Lectures, Cambridge University Press, Cambridge, 1995. MR1350074 (96k:35129)

[MS1] R. B. Melrose and J. Sjöstrand, Singularities of boundary value problems. I, Comm. Pure Appl. Math. 31 (1978), no. 5, 593-617. MR.0492794 (58 \#11859)

[MS2] R. B. Melrose and J. Sjöstrand, Singularities of boundary value problems. II, Comm. Pure Appl. Math. 35 (1982), no. 2, 129-168, DOI 10.1002/cpa.3160350202. MR644020 (83h:35120)

[NS] L. Noakes and L. Stoyanov, Travelling times in scattering by obstacles, Preprint 2014, arXiv:1404.4147 [math-ph].

[PS1] Vesselin M. Petkov and Luchezar N. Stoyanov, Geometry of reflecting rays and inverse spectral problems, Pure and Applied Mathematics (New York), John Wiley \& Sons, Ltd., Chichester, 1992. MR1172998 (93i:58161)

[PS2] Vesselin Petkov and Latchezar Stoyanov, Sojourn times of trapping rays and the behavior of the modified resolvent of the Laplacian (English, with English and French summaries), Ann. Inst. H. Poincaré Phys. Théor. 62 (1995), no. 1, 17-45. MR1313359 (96g:58199)

[Si1] Ya. Sinai, Dynamical systems with elastic reflections, Russian Math. Surveys 25 (1970), 137-190.

[Si2] Ya. Sinai, Development of Krylov's ideas, An addendum to the book: N.S.Krylov "Works on the foundations of statistical physics", Princeton Univ. Press, Princeton 1979, pp. 239281.

[SU] Plamen Stefanov and Gunther Uhlmann, Boundary rigidity and stability for generic simple metrics, J. Amer. Math. Soc. 18 (2005), no. 4, 975-1003, DOI 10.1090/S0894-0347-0500494-7. MR2163868(2006h:53031)

[SUV] P. Stefanov, G. Uhlmann and A. Vasy, Boundary rigidity with partial data, Preprint 2013, arXiv:1306.2995. 
[St1] Latchezar Stoyanov, Exponential instability for a class of dispersing billiards, Ergodic Theory Dynam. Systems 19 (1999), no. 1, 201-226, DOI 10.1017/S0143385799126543. MR1677157 (99m:58149)

[St2] Luchezar Stoyanov, On the scattering length spectrum for real analytic obstacles, J. Funct. Anal. 177 (2000), no. 2, 459-488, DOI 10.1006/jfan.2000.3645. MR.1795959 (2002b:35163)

[St3] Luchezar Stoyanov, Generalized Hamilton flow and Poisson relation for the scattering kernel (English, with English and French summaries), Ann. Sci. École Norm. Sup. (4) 33 (2000), no. 3, 361-382, DOI 10.1016/S0012-9593(00)00115-4. MR1775186 (2001g:37120)

[St4] Luchezar Stoyanov, Rigidity of the scattering length spectrum, Math. Ann. 324 (2002), no. 4, 743-771, DOI 10.1007/s00208-002-0358-9. MR.1942248 (2003j:58048)

[T] Michael E. Taylor, Grazing rays and reflection of singularities of solutions to wave equations, Comm. Pure Appl. Math. 29 (1976), no. 1, 1-38. MR0397175 (53 \#1035)

University of Western Australia, Crawley, Western Australia 6009, Australia

E-mail address: lyle.noakes@uwa.edu.au

University of Western Australia, Crawley, Western Australia 6009, Australia

E-mail address: luchezar.stoyanov@uwa.edu.au 